\title{
2D nanoporous membrane for cation removal from water: Effects of ionic valence, membrane hydrophobicity, and pore size
}

Cite as: J. Chem. Phys. 148, 222804 (2018); https://doi.org/10.1063/1.5013926

Submitted: 14 November 2017 . Accepted: 08 January 2018 . Published Online: 13 February 2018

Mateus Henrique Köhler (D), José Rafael Bordin (D), and Marcia C. Barbosa

\section{ARTICLES YOU MAY BE INTERESTED IN}

The influence of polarizability and charge transfer on specific ion effects in the dynamics of aqueous salt solutions

The Journal of Chemical Physics 148, 222803 (2018); https://doi.org/10.1063/1.5012682

Probing the Hofmeister series beyond water: Specific-ion effects in non-aqueous solvents The Journal of Chemical Physics 148, 222805 (2018); https://doi.org/10.1063/1.5017278

Molecular dynamics study of structure and vibrational spectra at zwitterionoic lipid/ aqueous $\mathrm{KCl}, \mathrm{NaCl}$, and $\mathrm{CaCl}_{2}$ solution interfaces

The Journal of Chemical Physics 148, 222801 (2018); https://doi.org/10.1063/1.5006543

The Journal

of Chemical Physics

The Emerging Investigators Special Collection and Awards Recognizing the excellent work of early career researchers! 


\title{
2D nanoporous membrane for cation removal from water: Effects of ionic valence, membrane hydrophobicity, and pore size
}

\author{
Mateus Henrique Köhler, ${ }^{1, a)}$ José Rafael Bordin,, $\left.{ }^{2, b}\right)$ and Marcia C. Barbosa ${ }^{1}$ \\ ${ }^{1}$ Instituto de Física, Universidade Federal do Rio Grande do Sul, Caixa Postal 15051, \\ 91501-970 Porto Alegre, Brazil \\ ${ }^{2}$ Campus Caçapava do Sul, Universidade Federal do Pampa, Av. Pedro Anunciação 111, \\ CEP 96570-000 Caçapava do Sul, Brazil
}

(Received 14 November 2017; accepted 8 January 2018; published online 13 February 2018)

Using molecular dynamic simulations, we show that single-layers of molybdenum disulfide $\left(\mathrm{MoS}_{2}\right)$ and graphene can effectively reject ions and allow high water permeability. Solutions of water and three cations with different valencies $\left(\mathrm{Na}^{+}, \mathrm{Zn}^{2+}\right.$, and $\left.\mathrm{Fe}^{3+}\right)$ were investigated in the presence of the two types of membranes, and the results indicate a high dependence of the ion rejection on the cation charge. The associative characteristic of ferric chloride leads to a high rate of ion rejection by both nanopores, while the monovalent sodium chloride induces lower rejection rates. Particularly, $\mathrm{MoS}_{2}$ shows $100 \%$ of $\mathrm{Fe}^{3+}$ rejection for all pore sizes and applied pressures. On the other hand, the water permeation does not vary with the cation valence, having dependence only with the nanopore geometric and chemical characteristics. This study helps us to understand the fluid transport through a nanoporous membrane, essential for the development of new technologies for the removal of pollutants from water. Published by AIP Publishing. https://doi.org/10.1063/1.5013926

\section{INTRODUCTION}

Centuries of misuse of natural resources have stressed available freshwater supplies throughout the world. With the rapid development of industries, chemical waste has been thrown deliberately into the water to the point of making it difficult to clean. Particularly, direct or indirect discharge of heavy metals into the environment has increased recently, especially in developing countries. ${ }^{1}$ Unlike organic contaminants, heavy metals are not biodegradable and tend to accumulate in living organisms. Many heavy metal ions are also known to be toxic or carcinogenic. ${ }^{2}$ Toxic heavy metals of particular concern in the treatment of industrial waste-water include zinc, copper, iron, mercury, cadmium, lead, and chromium.

As a result, the filtration process that can acquire freshwater from contaminated, brackish water or seawater is an effective method to also increase the potable water supply. Modern desalination is mainly based on reverse osmosis (RO) performed through membranes due to their low energy consumption and easy operation. Current RO plants have already been operated near the thermodynamic limit, with the applied pressure being only $10 \%-20 \%$ higher than the osmotic pressure of the concentrate. ${ }^{3}$ Meanwhile, advances in nanotechnology have inspired the design of novel membranes based on two-dimensional (2D) nanomaterials. Nanopores with diameters ranging from a few Angstroms to several nanometers can be drilled in membranes to fabricate molecular sieves. ${ }^{4}$ As the diameter of the pore approaches the size of the hydrated ions, various types of ions can be rejected by nanoporous

\footnotetext{
a)Electronic mail: mateus.kohler@ufrgs.br

b)Electronic mail: josebordin@unipampa.edu.br
}

membranes, leading to efficient water desalination. Graphene (Gra), a single-atom-thick carbon membrane, was demonstrated to have several orders of magnitude higher flux rates when compared with conventional zeolite membranes. ${ }^{5}$ In this way, graphene and graphene oxide are one of the most prominent materials for highly efficient membranes. ${ }^{6-8}$ More recently, other 2D materials have also been investigated for water filtration. A nanoporous single-layer of molybdenum disulfide $\left(\mathrm{MoS}_{2}\right)$ has shown a great desalination capacity. ${ }^{9-11}$ The possibility to craft the pore edge with Mo, S, or both provides flexibility to design the nanopore with desired functionality. In the same way, boron nitride nanosheets have also been investigated for water purification from distinct pollutants. ${ }^{12,13}$ Therefore, for the purpose of cleaning water, not only the nanopore size but also the hydrophobicity and the geometry of the porous membrane are considered.

For instance, the performance of the commercial RO membrane is usually on the order of $0.1 \mathrm{~L} / \mathrm{cm}^{2}$ day MPa $(1.18$ $\left.\mathrm{g} / \mathrm{m}^{2} \mathrm{~s} \mathrm{~atm}\right) .{ }^{14}$ With the aid of zeolite nanosheets, permeability as high as $1.3 \mathrm{~L} / \mathrm{cm}^{2}$ day MPa can be obtained. ${ }^{15}$ Recent studies have shown that $\mathrm{MoS}_{2}$ nanopore filters have the potential to achieve a water permeability of roughly $100 \mathrm{~g} / \mathrm{m}^{2} \mathrm{~s}$ $\mathrm{atm}^{10}-2$ orders of magnitude higher than the commercial RO. This is comparable with that measured experimentally for the graphene filter $\left(\sim 70 \mathrm{~g} / \mathrm{m}^{2} \mathrm{~s}\right.$ atm $)$ under similar conditions. ${ }^{16}$ These results have shown that the water permeability scales linearly with the pore density. Therefore, the water filtering performance of 2D nanopores can be even higher.

Controlling the size and shape of the pores created in these membranes, however, represents a huge experimental challenge. Inspired by a number of molecular dynamics (MD) studies predicting ultrahigh water permeability across graphene 
and other 2D nanoporous membranes, ${ }^{11,17}$ technologies have been developed to create and control the nanopore size and distribution. Methods including electron beam, ${ }^{18}$ ion irradiation, ${ }^{19}$ and chemical etching ${ }^{20}$ have been reported to introduce pores in graphene. Feng et al. ${ }^{21}$ have also developed a scalable method to controllably make nanopores in single-layer $\mathrm{MoS}_{2}$ with subnanometer precision using the electrochemical reaction (ECR). Recently, Liu and colleagues ${ }^{22}$ investigated the geometrical effect of the nanopore shape on ionic blockage induced by DNA translocation through h-BN and $\mathrm{MoS}_{2}$ nanopores. They observed a geometry-dependent ion scattering effect and further proposed a modified ionic blockage model which is highly related to the ionic profile caused by geometrical variations. Additionally, recent experimental efforts have been devoted to amplify the filtering efficiency of the nanoporous membranes. Wang and colleagues ${ }^{23}$ mechanistically related the performance of $\mathrm{MoS}_{2}$ membranes to the size of their nanochannels in different hydration states. They attributed the high water flux (30-250 L/m $\mathrm{m}^{2} \mathrm{~h}$ bars) of $\mathrm{MoS}_{2}$ membranes to the low hydraulic resistance of the smooth, rigid $\mathrm{MoS}_{2}$ nanochannels. The membrane compaction with high pressure has also been found to create a neatly stacked nanostructure with minimum voids, leading to stable water flux and enhanced separation performance. By tuning the pore creation process, Jang et al. ${ }^{24}$ have demonstrated nanofiltration membranes that reject small molecules but offer high permeance to water or monovalent ions. Also, studies have shown how defects, oxidation, and functionalization can affect the ionic blockage ${ }^{25-27}$ All of these studies point to a near future where 2D membranes will have a major impact on desalination processes.

In this work, we address the issue of the selectivity of the porous membrane. In order to do that, we compare the water filtration capacity of $\mathrm{MoS}_{2}$ and graphene through molecular dynamics simulations. While graphene is a purely hydrophobic material, $\mathrm{MoS}_{2}$ sheets have both hydrophobic (S) and hydrophilic (Mo) sites. Recent studies have shown that the water dynamics and structure inside hydrophobic or hydrophilic pores can be quite distinct regarding the pore size $^{28-30}$ and even near hydrophobic or hydrophilic protein sites. ${ }^{31}$ Three cations are considered: the standard monovalent sodium $\left(\mathrm{Na}^{+}\right)$, the divalent zinc $\left(\mathrm{Zn}^{2+}\right)$, and the trivalent iron $\left(\mathrm{Fe}^{3+}\right)$. The study of sodium removal is relevant due to its applications for water desalination. ${ }^{32-34}$ Zinc is a trace element that is essential for human health. It is important for the physiological functions of living tissue and regulates many biochemical processes. However, excess of zinc can cause eminent health problems. ${ }^{35}$ The cation $\mathrm{Zn}^{2+}$ is ranked 75th in the Comprehensive Environmental Response, Compensation and Liability Act (CERCLA) 2017 priority list of hazardous substances. In its trivalent form, ferric chloride $\mathrm{Fe}^{3+} \mathrm{Cl}_{3}^{-}$is a natural flocculant, with high power of aggregation. It is also on the CERCLA list with recommended limit concentration of $0.3 \mathrm{mg} / \mathrm{l}$. In this way, we explore the water permeation and cations rejection by nanopore with distinct radii. Our results show that the hydrophilic/hydrophobic $\mathrm{MoS}_{2}$ nanopores have a higher salt rejection in all scenarios, while the purely hydrophobic graphene nanopores have a higher water permeation. Especially, $\mathrm{MoS}_{2}$ membranes show the impressive capacity of blocking all the trivalent iron cations regardless of the nanopore size.

Our paper is organized as follows. In Sec. II, we introduce our model and the details about the simulation method. In Sec. III, we show and discuss our results for the water permeation in the distinct membranes, while in Sec. IV, we show the ion rejection properties for each case. Finally, a summary of our results and the conclusions are shown in Sec. V.

\section{COMPUTATIONAL DETAILS AND METHODS}

Molecular dynamics (MD) simulations were performed using the Large-scale Atomic/Molecular Massively Parallel Simulator (LAMMPS) package. ${ }^{36}$ A typical simulation box consists of a graphene sheet acting as a rigid piston in order to apply an external force (pressure) over the ionic solution. The pressure gradient forces the solution against the 2D nanopore:

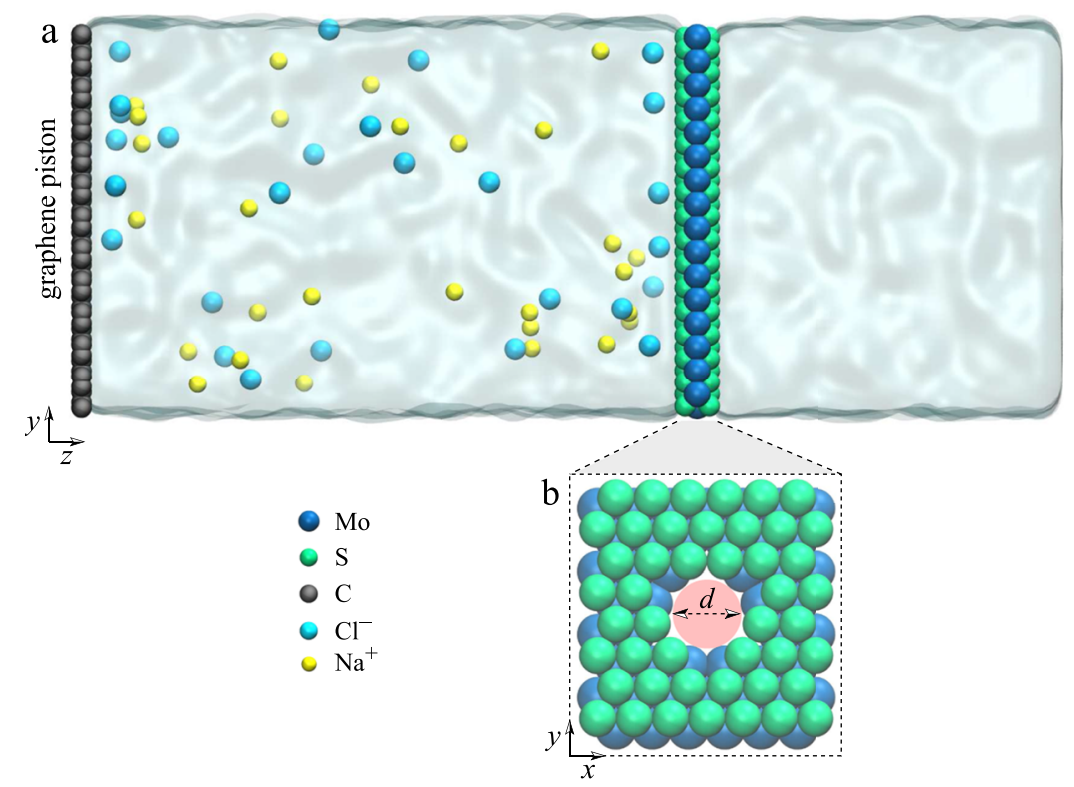

FIG. 1. (a) Schematic representation of the simulation framework. The system is divided as follows: On the left side we can see the piston (graphene) pressing the ionic solution (in this case, water $+\mathrm{NaCl}$ ) against the $\mathrm{MoS}_{2}$ nanopore. For the case of a graphene nanopore, the depiction is the same, but with a porous graphene sheet instead of the $\mathrm{MoS}_{2}$ sheet. On the right side, we have bulk water. (b) Definition of the pore diameter $d$. 
a single-layer of molybdenum disulfide or graphene. Figure 1 shows the schematic representation of the simulation framework.

A nanopore was drilled in both $\mathrm{MoS}_{2}$ and graphene sheets by removing the desired atoms, as shown in Fig. 1. The accessible pore diameters considered in this work range from 0.26 to $0.95 \mathrm{~nm}$ for the $\mathrm{MoS}_{2}$ (which means a pore area ranging from 5.5 to $71 \AA^{2}$ ) and $0.17-0.92 \mathrm{~nm}$ for the graphene (with area ranging from 2.5 to $67 \AA^{2}$ ). M. Heiranian et al. ${ }^{11}$ have studied different $\mathrm{MoS}_{2}$ nanopore compositions for water filtration: with only Mo, only S, and a mix of the two atoms at the pore's edge. They found similar ion rejection rates for both cases. Here, in order to account for circular nanopores, mixed pore edges have been chosen. The system contains 22000 atoms distributed in a box with dimensions $5 \times 5$ $\times 13 \mathrm{~nm}$ in $\mathrm{x}, \mathrm{y}$, and $\mathrm{z}$, respectively. Although the usual salinity of seawater is $\sim 0.6 \mathrm{M}$, we choose a molarity of $\sim 1.0 \mathrm{M}$ for all the cations $\left(\mathrm{Na}^{+}, \mathrm{Zn}^{2+}\right.$, and $\left.\mathrm{Fe}^{3+}\right)$ due to the computational cost associated with low-molarity solutions.

The TIP4P $/ 2005^{37}$ water model was used and the SHAKE algorithm ${ }^{38}$ was employed to maintain the rigidity of the water molecules. The non-bonded interactions are described by the Lennard-Jones (LJ) potential with a cutoff distance of $0.1 \mathrm{~nm}$ and the parameters tabulated in Table I. The Lorentz-Berthelot mixing rule was used to obtain the $\mathrm{LJ}$ parameters for different atomic species. The long-range electrostatic interactions were calculated by the particle-particle-particle mesh method. ${ }^{39}$ Periodic boundary conditions were applied in all the three directions.

For each simulation, the system was first equilibrated for constant number of particles, pressure, and temperature (NPT) ensemble for $1 \mathrm{~ns}$ at $\mathrm{P}=1 \mathrm{~atm}$ and $\mathrm{T}=300 \mathrm{~K}$. Graphene and $\mathrm{MoS}_{2}$ atoms were held fixed in the space during equilibration, and the NPT simulations allow water to reach its equilibrium density $\left(1 \mathrm{~g} / \mathrm{cm}^{3}\right)$. After the pressure equilibration, a 5 ns simulation was carried out in the constant number of particles, volume, and temperature (NVT) ensemble to further equilibrate the system at the same $\mathrm{T}=300 \mathrm{~K}$. Finally, a $10 \mathrm{~ns}$ production run was carried out, also in the NVT ensemble. The Nosé-Hoover thermostat ${ }^{44,45}$ was used at each 0.1 ps in both NPT and NVT simulations, and the Nosé-Hoover barostat was used to keep the pressure constant in the NPT simulations. Different external pressures were applied on the rigid piston to characterize the water filtration through the 2D (graphene and $\mathrm{MoS}_{2}$ ) nanopores. For simplicity, the pores

TABLE I. The Lennard-Jones parameters and charges of the simulated atoms. The crossed parameters were obtained by Lorentz-Berthelot rule.

\begin{tabular}{|c|c|c|c|}
\hline Interaction & $\sigma(\mathrm{nm})$ & $\varepsilon(\mathrm{kcal} / \mathrm{mol})$ & Charge \\
\hline $\mathrm{C}-\mathrm{C}^{40}$ & 3.39 & 0.0692 & 0.00 \\
\hline Mo-Mo ${ }^{41}$ & 4.20 & 0.0135 & 0.60 \\
\hline$S-S^{41}$ & 3.13 & 0.4612 & -0.30 \\
\hline $\mathrm{O}-\mathrm{O}^{37}$ & 3.1589 & 0.1852 & -1.1128 \\
\hline $\mathrm{H}-\mathrm{H}$ & 0.00 & 0.00 & 0.5564 \\
\hline $\mathrm{Na}-\mathrm{Na}^{42}$ & 2.52 & 0.0347 & 1.00 \\
\hline $\mathrm{Cl}-\mathrm{Cl}^{42}$ & 3.85 & 0.3824 & -1.00 \\
\hline $\mathrm{Zn}-\mathrm{Zn}^{43}$ & 0.0125 & 1.960 & 2.00 \\
\hline $\mathrm{Fe}-\mathrm{Fe}^{43}$ & 0.18 & 0.745 & 3.00 \\
\hline
\end{tabular}

were held fixed in space to study solely the water transport and ion rejection properties of these materials. The external pressures range from 10 to $100 \mathrm{MPa}$. These are higher than the osmotic pressure used in the experiments. The reason for applying such high pressures at MD simulations with running time in nanosecond scale is that the low pressures would yield a very low water flux that would not go above the statistical error. We carried out three independent simulations for each system collecting the trajectories of atoms every picosecond.

\section{WATER FLUX}

First, let us compare the flux performance of the graphene and the $\mathrm{MoS}_{2}$ membranes. In Fig. 2, we show the water flux through 2D nanopores in number of molecules per nanosecond $\left(\mathrm{MoS}_{2}\right.$ and graphene) as a function of the applied pressure gradient for different pore diameters. The water is filtered from a reservoir containing an ionic solution of either monovalent sodium $\left(\mathrm{Na}^{+}\right)$, divalent zinc $\left(\mathrm{Zn}^{2+}\right)$, or trivalent iron cations $\left(\mathrm{Fe}^{3+}\right)$. In all cases, chlorine $\left(\mathrm{Cl}^{-}\right)$was used as the standard anion. Four pore sizes for each material were investigated.

Our results indicate that for the smaller pore diameter, the black points in Fig. 2, both materials have the same water permeation. However, for the other values of pore diameter, the graphene membrane shows a higher water flux, for all applied pressure gradient. While the flux at the purely hydrophobic graphene pore for a fixed pressure monotonically increases with the pore diameter, this is not the case for the $\mathrm{MoS}_{2}$ pore for which the flows show a minimum pore diameter of around $0.37 \mathrm{~nm}$ probably due to the nonuniform distribution of the hydrophobic and hydrophilic sites of the pore. Figures 2(a)2(c) show that this behavior of the water flux is not affected by the cation valence, only by the applied pressure, by geometric effects, and by the pore composition. For instance, the $0.46 \mathrm{~nm}$ graphene pore shows enhanced water flux compatible with the $0.6 \mathrm{~nm} \mathrm{MoS} 2$ pore for all cations. Therefore, it is clear that pore composition affects the water permeation properties more than the water-ion interaction.

This result agrees with the findings by Aluru and his group ${ }^{11}$ where they showed that even a small change in pore composition can lead to enhanced water flux through a $\mathrm{MoS}_{2}$ nanocavity. This is also consistent with our recent findings that the dynamics of water inside nanopores with diameter $\approx 1.0 \mathrm{~nm}$ is strongly affected by the presence of hydrophilic or hydrophobic sites. ${ }^{29}$ This investigation, over distinct cation valences and membranes, highlights the importance of the nanopore physical-chemistry properties for water filtration processes.

To quantify the water permeability through the pores, we compute the permeability coefficient, $p$, across the pore. For dilute solutions,

$$
p=\frac{j_{\mathrm{w}}}{-V_{\mathrm{w}} \Delta C_{\mathrm{s}}+\frac{V_{\mathrm{W}}}{N_{\mathrm{A}} k_{\mathrm{B}} T} \Delta P},
$$

where $j_{\mathrm{w}}$ is the flux of water $\left(\mathrm{H}_{2} \mathrm{O} / \mathrm{ns}\right), V_{\mathrm{w}}$ is the molar volume of water $(19 \mathrm{ml} / \mathrm{mol}), \Delta C_{\mathrm{s}}$ is the concentration gradient of the solute (1.0M), $N_{A}$ is the Avogadro number, $k_{\mathrm{B}}$ is the Boltzmann 

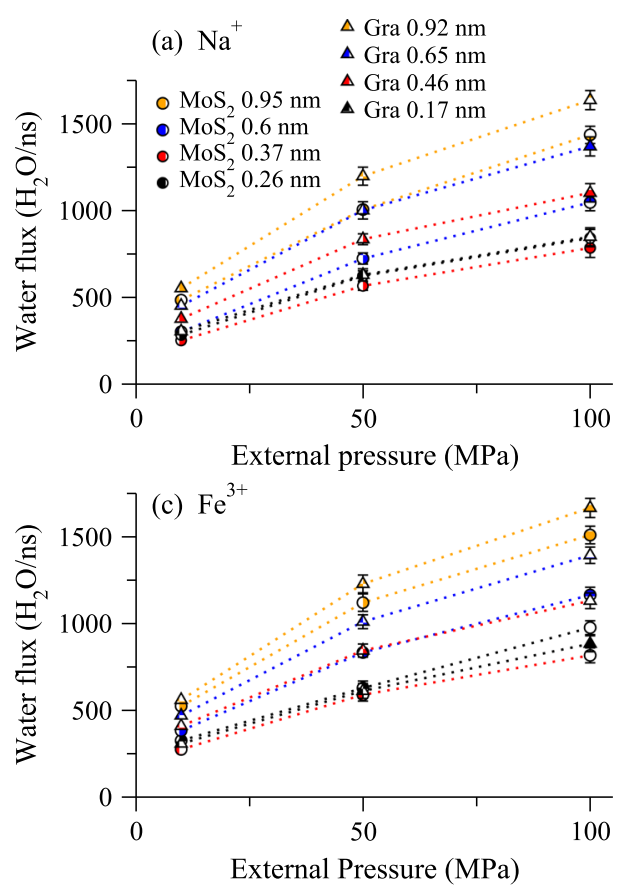
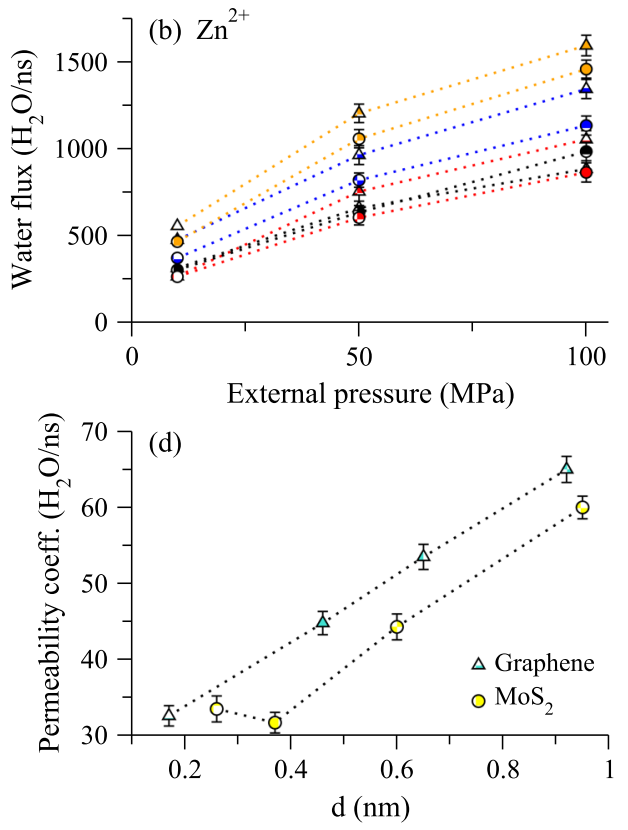

FIG. 2. Water flux as a function of the applied pressure for $\mathrm{MoS}_{2}$ and graphene nanopores with similar pore areas. (a) Monovalent $\mathrm{Na}^{+}$, (b) divalent $\mathrm{Zn}^{2+}$, and (c) trivalent $\mathrm{Fe}^{3+}$ cations are considered for the ionic solution at the reservoir. (d) Water permeability through the pores as a function of the pore diameter for the case of $\Delta \mathrm{P}=50 \mathrm{MPa}$. The dotted lines are a guide to the eye. constant, $T$ is the temperature $(300 \mathrm{~K})$, and $\Delta \mathrm{P}$ is the applied hydrodynamic pressure $(\mathrm{MPa})$.

The case of $\Delta \mathrm{P}=50 \mathrm{MPa}$ is shown in Fig. 2(d). The permeability coefficient of the $\mathrm{MoS}_{2}$ ranges from approximately 33 to $55 \mathrm{H}_{2} \mathrm{O} / \mathrm{ns}$ for the 0.26 and $0.95 \mathrm{~nm}$ diameters, respectively. The graphene nanopore presents a permeability coefficient of $\sim 34-63 \mathrm{H}_{2} \mathrm{O} / \mathrm{ns}$ as the pore diameter is varied from 0.17 to $0.92 \mathrm{~nm}$, respectively. For smaller pores, the difference between $\mathrm{MoS}_{2}$ and graphene is inside the error bars, whereas for the larger pores, both materials exhibit high permeability rates, with a slight advantage in the case of graphene.

The water structure and dynamics inside nanopores are strongly related. ${ }^{29,46}$ Therefore, distinct structural regimes can lead to different diffusive behaviors. In Fig. 3, we present the distribution of water molecules in the z-direction inside the $\mathrm{MoS}_{2}$ (solid line) and graphene (dotted line) nanopores. As for the water flux, the water axial distribution is not affected by the cation valence. Therefore, for simplicity and since there are more studies about monovalent salts, we show only the $\mathrm{Na}^{+}$case. The nanopore length in the $\mathrm{z}$-direction, considering the van der Waals diameter for each sheet, is $0.63(-0.315-$ $0.315) \mathrm{nm}$ for the $\mathrm{MoS}_{2}$ and $0.34(-0.17-0.17) \mathrm{nm}$ for the graphene. The structures inside both pores are considerably different. For the graphene nanopore, shown in Fig. 3(a), there is no favorable positions for the water molecules to remain throughout the simulation. This can be related to the hydrophobic characteristic of the graphene sheet and the high slippage observed for water inside carbon nanopores. ${ }^{47,48}$ Since all the pore is hydrophobic, there is no preferable position for the water molecules, and the permeability is higher. On the other hand, along the $\mathrm{MoS}_{2}$ cavity we can observe a high structuration in three sharp peaks, as shown in Fig. 3(b). This structuration comes from the existence of hydrophilic (Mo) and hydrophobic sites ( $\mathrm{S}$ atoms). This layered organization within the $\mathrm{MoS}_{2}$ nanopore can be linked to the reduced flux compared with graphene, since it implies an additional term in the energy required for the water molecule to pass through the pore.

The higher water flux through graphene nanopores compared with $\mathrm{MoS}_{2}$ implies that for a desired water flux, a smaller applied pressure is needed. Nevertheless, it is important to note that both fluxes are higher, especially when compared with current desalination technologies. ${ }^{11,49}$ Therefore, both materials are capable of providing a high water permeability. The question is whether these materials are also able to effectively clean the water by removing the ions.
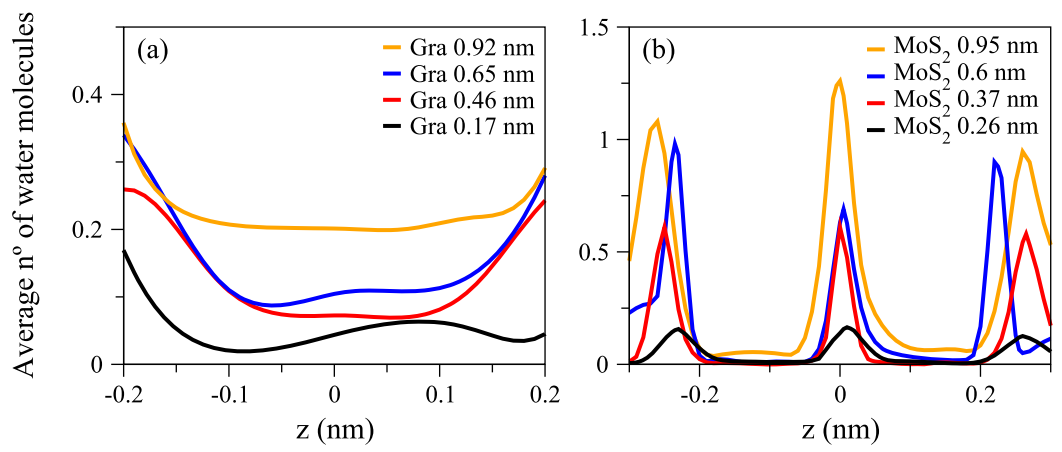

FIG. 3. Averaged axial distribution of water molecules inside the (a) graphene (Gra) and (b) $\mathrm{MoS}_{2}$ nanopores with distinct diameters. Here, $\mathrm{z}=0$ is at the center of the pore, the external pressure is $\Delta \mathrm{P}=10 \mathrm{MPa}$, and the cation is the $\mathrm{Na}^{+}$. 


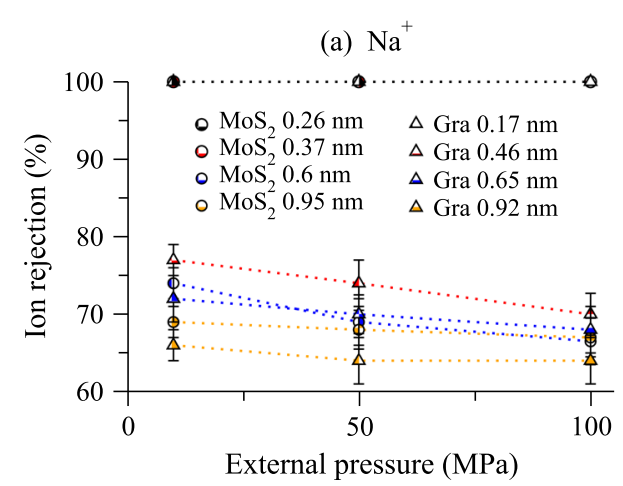

(a) $\mathrm{Na}^{+}$

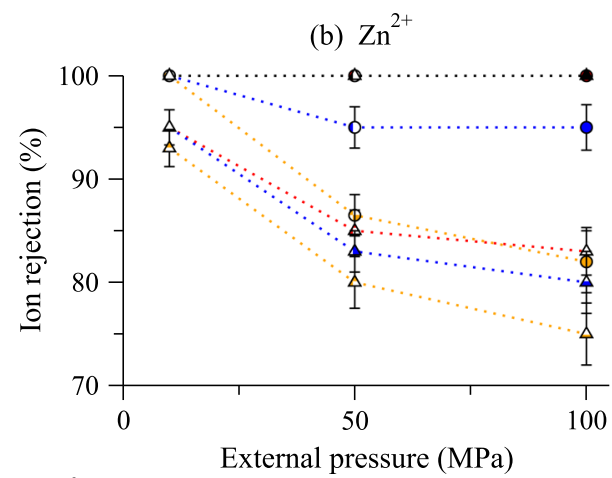

(c) $\mathrm{Fe}^{3+}$

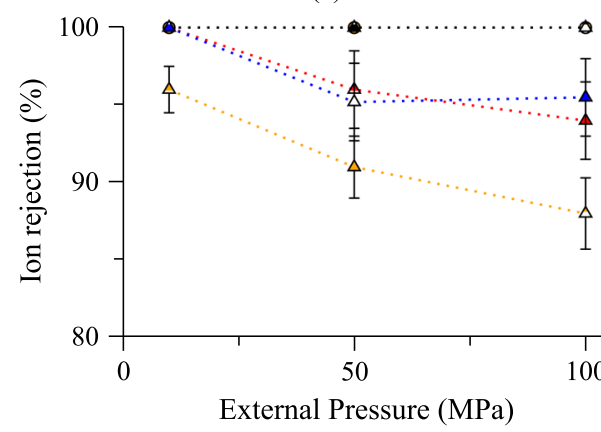

FIG. 4. Percentage of ion rejection by various pores as a function of the applied pressure. Pores with different diameters are considered.

\section{ION REJECTION EFFICIENCY}

The other important aspect for the cleaning of water is the membrane's ability to separate water and ions. In this way, we investigate how the cation valence and the pore size affect the percentage of rejected ions. In Fig. 4, we show the percentage of total ions rejected by the 2D nanopores as a function of the applied pressure for the three cations. The pore diameters are the same as discussed in Sec. III.

The ion rejection by the smallest pores, 0.17 and $0.26 \mathrm{~nm}$ for graphene and $\mathrm{MoS}_{2}$, respectively, was $100 \%$ for all applied pressures and cation solutions. This is expected since the a
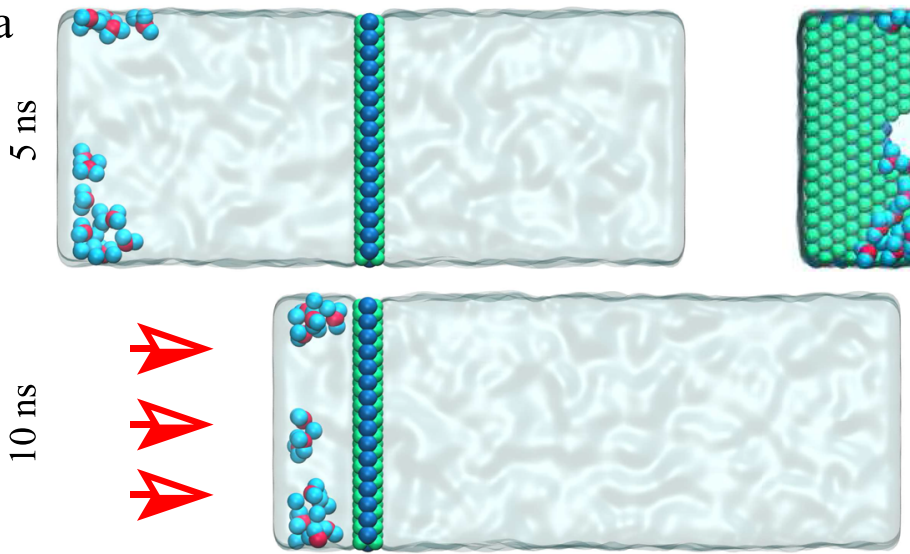

b

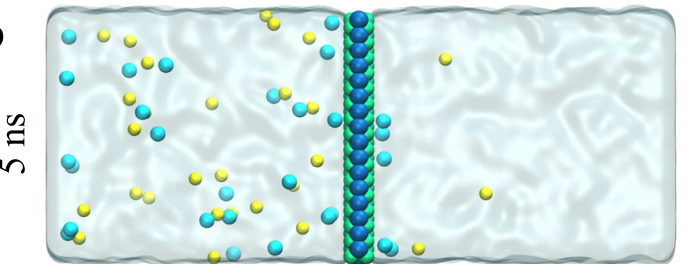

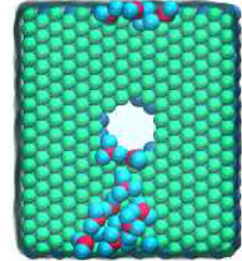

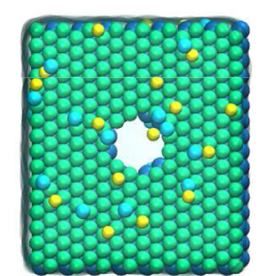

FIG. 5. Side and front view snapshots of (a) $\mathrm{Fe}^{3+} \mathrm{Cl}^{-}$ cluster formation preventing the ion passage through a $0.95 \mathrm{~nm} \mathrm{MoS} 2$ nanopore and (b) monovalent $\mathrm{Na}^{+} \mathrm{Cl}^{-}$ passing through the same nanopore without clusterization for an external applied pressure of $50 \mathrm{MPa}$. 
pore size is much smaller than the hydration radii of the cations. Therefore, it is more energetically favorable for the cation to remain in the bulk solution instead of stripping off the water and entering the pore. ${ }^{50}$ As the pore diameter increases, this energetic penalty becomes smaller. In addition, the valence plays a crucial role here, with the monovalent ions having a smaller penalty than divalent and trivalent cations. In this way, for the nanopores with diameters $0.37 \mathrm{~nm}$ and $0.46 \mathrm{~nm}$ for graphene and $\mathrm{MoS}_{2}$, respectively, $\mathrm{Na}^{+}$and $\mathrm{Cl}^{-}$ions flow through the pore reducing the rejection efficiency for both materials, as we can see in Fig. 4(a). However, it is important to note that the ion rejection performance of molybdenum disulfide membranes is superior to that observed for graphene membranes for all ranges of pressure, sizes, and cation valences. For instance, for the divalent case $\mathrm{Zn}^{2+}$, shown in Fig. 4(b), and the smaller $\Delta \mathrm{P}$, the rejection is $100 \%$ for all pore sizes in the $\mathrm{MoS}_{2}$ membrane, while for the graphene membrane, we observe cation permeation for the bigger pores.

The $\mathrm{MoS}_{2}$ membrane shows a very good performance for the rejection of the trivalent cation $\mathrm{Fe}^{3+}$. As Fig. 4(c) shows, for all nanopore sizes and applied pressures the rejection is $100 \%$. Such efficiency was not observed in the graphene membranes, were only the case with small pore diameter as $100 \%$ of iron rejection. Here, we should address that not only the hydration shell plays an important role in the cations rejection. While sodium chloride is uniformly dispersed in water and we do not observe clusters at the simulated concentration, the iron cations tend to form large clusters of ferric chlorides in solution, as shown in Fig. 5. Moreover, we observe these structures throughout the whole simulation, and even at high-pressure regime the clusters remain too large to overcome the pore. In fact, ferric chlorides are effective as primary coagulants due to their associative character in solution. At controlled concentrations, it is excellent for both drinking and wastewater treatment applications, including phosphorus removal, ${ }^{51}$ sludge conditioning, and struvite control. ${ }^{52,53}$ It also prevents odor and corrosion by controlling hydrogen sulfide formation. Additionally, our results indicate that the associative properties of ferric chlorides can be used to increase the efficiency of salt rejection by both $\mathrm{MoS}_{2}$ and graphene nanopores, which may contribute in water cleaning devices.

\section{SUMMARY AND CONCLUSIONS}

We have calculated water fluxes through various $\mathrm{MoS}_{2}$ and graphene nanopores and the respective percentage of the total ions rejected by both materials as a function of the applied pressure gradient. Our results indicate that 2D nanoporous membranes are promising for water purification and salt rejection. The selectivity of the membranes was found to depend on factors such as the pore diameter, the cationic valence, and the applied pressure. Nevertheless, our results show that the ion valency does not affect the water permeation-it is only affected by the pore size and chemical composition.

Particularly, our findings indicate that graphene is a better water conductor than $\mathrm{MoS}_{2}$, with a higher permeability coefficient, although both materials have presented high water fluxes. On the other hand, $\mathrm{MoS}_{2}$ nanopores with water-accessible pore diameters ranging from 0.26 to $0.95 \mathrm{~nm}$ strongly reject ions even at theoretically high pressures of 100 $\mathrm{MPa}$. Additionally, the rejection is shown to depend strongly on the ion valence. It reaches $100 \%$ for trivalent ferric chloride $\left(\mathrm{Fe}^{3+} \mathrm{Cl}_{3}^{-}\right)$for all $\mathrm{MoS}_{2}$ pore sizes and applied pressures. This is a direct result of the ability of heavy metals to form agglomerates, eventually exhibiting long ionic chains. At the same time, this did not affect the water flux. Then, the ferric chloride properties can be used to improve the effectiveness of 2D material-based nanofilters. New studies are performed in this direction.

\section{ACKNOWLEDGMENTS}

We thank the Brazilian agencies CNPq and INCT-FCx for the financial support and CENAPAD/SP and CESUP/UFRGS for the computer time.

${ }^{1}$ D. Ko, J. S. Lee, H. A. Patel, M. H. Jakobsen, Y. Hwange, C. T. Yavuzan, H. B. Hansen, and H. R. Andersen, J. Hazard. Mater. 332, 140 (2017).

${ }^{2}$ M. B. Gumpu, S. Sethuraman, U. M. Krishnan, and J. B. B. Rayappan, Sens. Actuators, B 213, 515 (2015).

${ }^{3} \mathrm{M}$. Li, Desalination 422, 124 (2017).

${ }^{4}$ L. Wang, M. S. H. Boutilier, P. R. Kidambi, D. Jang, N. G. Hadjiconstantinou, and R. Karnik, Nat. Nanotechnol. 12, 509 (2017).

${ }^{5}$ K. Celebi, J. Buchheim, R. M. Wyss, A. Droudian, P. Gasser, I. Shorubalko, J.-I. Kye, C. Lee, and H. G. Park, Science 344, 289 (2014).

${ }^{6}$ Q. Xu, H. Xu, J. Chen, Y. Lv, C. Dong, and T. S. Sreeprasadc, Inorg. Chem. Front. 2, 417 (2015).

${ }^{7}$ K. C. Kemp, H. Seema, M. Saleh, N. H. Le, K. Mahesh, V. Chandra, and K. S. Kim, Nanoscale 5, 3149 (2013).

${ }^{8}$ L. Huang, M. Zhang, C. Li, and G. Shi, J. Phys. Chem. Lett. 6, 2806 (2015).

${ }^{9}$ J. Kou, J. Yao, L. Wu, X. Zhou, H. Lu, F. Wu, and J. Fan, Phys. Chem. Chem. Phys. 18, 22210 (2016).

${ }^{10}$ W. Li, Y. Yang, J. K. Weber, G. Zhang, and R. Zhou, ACS Nano 10, 1829 (2016).

${ }^{11}$ M. Heiranian, A. B. Farimani, and N. R. Aluru, Nat. Commun. 6, 8616 (2015).

${ }^{12}$ W. Lei, D. Portehault, D. Liu, S. Qin, and Y. Chen, Nat. Commun. 4, 1777 (2013).

${ }^{13}$ J. Azamat, B. S. Sattary, A. Khataee, and S. W. Joo, J. Mol. Graphics Modell. 61, 13 (2015).

${ }^{14}$ M. M. Pendergast and E. M. Hoek, Energy Environ. Sci. 4, 1946 (2011).

${ }^{15}$ S. H. Jamali, T. J. H. Vlugt, and L.-C. Lin, J. Phys. Chem. C 121, 11273 (2017).

${ }^{16}$ S. P. Surwade, S. N. Smirnov, I. V. Vlassiouk, R. R. Unocic, G. M. Veith, S. Dai, and S. M. Mahurin, Nat. Nanotechnol. 10, 459 (2015).

${ }^{17}$ D. Cohen-Tanugi and J. C. Grossman, Nano Lett. 12, 3602 (2012).

${ }^{18}$ S. Garaj, W. Hubbard, A. Reina, J. Kong, D. Branton, and J. A. Golovchenko, Nature 467, 190 (2010).

${ }^{19}$ K. Yoon, A. Rahnamoun, J. L. Swett, V. Iberi, D. A. Cullen, I. V. Vlassiouk, A. Belianinov, S. Jesse, X. Sang, O. S. Ovchinnikova, A. J. Rondinone, R. R. Unocic, and A. C. van Duin, ACS Nano 10, 8376 (2016).

${ }^{20}$ S. C. OHern, D. Jang, S. Bose, J.-C. Idrobo, Y. Song, T. Laoui, J. Kong, and R. Karnik, Nano Lett. 15, 3254 (2015).

${ }^{21}$ J. Feng, K. Liu, M. Graf, M. Lihter, R. D. Bulushev, D. Dumcenco, D. T. L. Alexander, D. Krasnozhon, T. Vuletic, A. Kis, and A. Radenovic, Nano Lett. 15, 3431 (2015).

${ }^{22}$ K. Liu, M. Lihter, A. Sarathy, S. Caneva, H. Qiu, D. Deiana, V. Tileli, D. T. L. Alexander, S. Hofmann, D. Dumcenco, A. Kis, J.-P. Leburton, and A. Radenovic, Nano Lett. 17, 4223 (2017).

${ }^{23}$ Z. Wang, Q. Tu, S. Zheng, J. J. Urban, S. Li, and B. Mi, Nano Lett. 17, 7289 (2017).

${ }^{24}$ D. Jang, J.-C. Idrobo, T. Laoui, and R. Karnik, ACS Nano 11, 10042 (2017).

${ }^{25}$ J. L. Achtyl, R. R. Unocic, L. Xu, Y. Cai, M. Raju, W. Zhang, R. L. Sacci, I. V. Vlassiouk, P. F. Fulvio, P. Ganesh, D. J. Wesolowski, S. Dai, A. C. T. van Duin, M. Neurock, and F. M. Geiger, Nat. Commun. 6, 6539 (2015). 
${ }^{26}$ G. Levita, P. Restuccia, and M. Righi, Carbon 107, 878 (2016).

${ }^{27}$ J. Abrahamand, K. S. Vasu, C. D. Williams, K. Gopinadhan, Y. Su, C. T. Cherian, J. Dix, E. Prestat, S. J. Haigh, I. V. Grigorieva, P. Carbone, A. K. Geim, and R. R. Nair, Nat. Nanotechnol. 12, 546 (2017).

${ }^{28}$ I. Moskowitz, M. A. Snyder, and J. Mittal, J. Chem. Phys. 141, 18 C532 (2014).

${ }^{29}$ M. H. Köhler, J. R. Bordin, L. B. da Silva, and M. C. Barbosa, Phys. Chem. Chem. Phys. 19, 12921 (2017).

${ }^{30}$ J. R. Bordin and M. C. Barbosa, Phys. A: Stat. Mech. Appl. 467, 137 (2017).

${ }^{31}$ M. H. Köhler, R. C. Barbosa, L. B. da Silva, and M. C. Barbosa, Phys. A: Stat. Mech. Appl. 468, 733 (2017).

${ }^{32}$ B. Corry, J. Phys. Chem. B 112, 1427 (2008).

${ }^{33}$ R. Das, M. E. Ali, S. B. A. Hamid, S. Ramakrishna, and Z. Z. Chowdhury, Desalination 336, 97 (2014).

${ }^{34}$ K. A. Mahmoud, B. Mansoor, A. Mansour, and M. Khraisheh, Desalination 356, 208 (2015).

${ }^{35}$ F. Fu and Q. Wang, J. Environ. Manage. 92, 407 (2011).

${ }^{36}$ S. Plimpton, J. Comput. Phys. 117, 1 (1995).

${ }^{37}$ J. Abascal and C. Vega, J. Chem. Phys. 123, 234505 (2005).

${ }^{38}$ J. P. Ryckaert, G. Ciccotti, and H. J. C. Berendsen, J. Comput. Phys. 23, 327 (1977)
${ }^{39}$ R. W. Hockney and J. W. Eastwood, Computer Simulation Using Particles (McGraw-Hill, New York, 1981).

${ }^{40}$ A. Barati Farimani and N. R. Aluru, J. Phys. Chem. B 115, 12145 (2011).

${ }^{41}$ T. Liang, S. R. Phillpot, and S. B. Sinnott, Phys. Rev. B 79, 245110 (2009).

${ }^{42}$ R. Fuentes-Azcatl and M. C. Barbosa, J. Phys. Chem. B 120, 2460 (2016).

${ }^{43}$ K. R. Hinkle, C. J. Jameson, and S. Murad, J. Chem. Eng. Data 61, 1578 (2016)

${ }^{44}$ S. Nosé, Mol. Phys. 52, 255 (1984).

${ }^{45}$ W. G. Hoover, Phys. Rev. A 31, 1695 (1985).

${ }^{46}$ J. R. Bordin, A. Diehl, and M. C. Barbosa, J. Phys. Chem. B 117, 7047 (2013).

${ }^{47}$ K. Falk, F. Sedlmeier, L. Joly, R. R. Netz, and L. Bocquet, Nano Lett. 10, 4067 (2010).

${ }^{48}$ G. Tocci, L. Joly, and A. Michaelides, Nano Lett. 14, 6872 (2014).

${ }^{49}$ J. Azamat and A. Khataee, Comput. Mater. Sci. 137, 201 (2017).

${ }^{50}$ J. R. Bordin, A. Diehl, M. C. Barbosa, and Y. Levin, Phys. Rev. E 85, 031914 (2012).

${ }^{51}$ B. Kim, M. Gautier, P. Molle, P. Michel, and R. Gourdon, Ecol. Eng. 80, 53 (2015).

${ }^{52}$ O. Amuda and I. Amoo, J. Hazard. Mater. 141, 778 (2007).

${ }^{53}$ J. Sun, I. Pikaar, K. R. Sharma, J. Keller, and Z. Yuan, Water Res. 71, 150 (2015). 